\title{
Synoptic Analysis of Epidemiologic Evidence of Glioma Risk from Mobile Phones
}

\author{
Norbert Leitgeb \\ Institute of Health Care Engineering, Graz University of Technology, Graz, Austria \\ Email: norbert.leitgeb@tugraz.at
}

Received 15 August 2015; accepted 26 September 2015; published 29 September 2015

Copyright (C) 2015 by author and Scientific Research Publishing Inc.

This work is licensed under the Creative Commons Attribution International License (CC BY).

http://creativecommons.org/licenses/by/4.0/

c) (i) Open Access

\begin{abstract}
Concern about brain cancer risks from mobile phone use, in particular of glioma, is mainly based on epidemiologic studies reporting on an increased risk estimate (odds ratio, OR) in particular of long-term and/or heavy users. Concern is enhanced by contradictory results and alarming conclusions from some epidemiologic studies. In a new synoptic approach all reported data from epidemiological studies published since 2001 were analyzed. This approach provided new insight with regard to the suspected link between mobile phone use and glioma. Two quite different data pools could be identified with numerous studies from one single research group opposing all other national and international studies. It could be shown that in spite of puzzling differences of their data pattern, in dependence of the number of exposed cases both data pools exhibit the similar trend of ORs towards reduced glioma risk with increasing statistical power, finally even converging towards a reduced risk, although from either side of the zero-risk line. While in the pooled data a potential long-term risk could be masked by reassuring short-term data the synoptic analysis in dependence of dose surrogates such as cumulated use time, cumulated call time or cumulated number of calls the seemingly increased long-term risk of glioma could be identified as an effect from low statistical power. In spite of worrying differences among some epidemiological studies, overall, the synoptic analysis of the entire body of data supports reassuring rather than alarming conclusions on glioma risks from mobile phone use even in long-term and/or heavy users.
\end{abstract}

\section{Keywords}

Health Risk, Dose Response, Carcinogenicity, Electromagnetic Field, Telecommunication

\section{Introduction}

Based on epidemiologic studies mainly at mobile phone frequencies around 1 - $2 \mathrm{GHz}$ the International Agency

How to cite this paper: Leitgeb, N. (2015) Synoptic Analysis of Epidemiologic Evidence of Glioma Risk from Mobile Phones. Journal of Electromagnetic Analysis and Applications, 7, 233-243. http://dx.doi.org/10.4236/jemaa.2015.79025 
for Research on Cancer (IARC) of the World Health Organization (WHO) has concluded there was "limited evidence" that radio frequency (RF) electromagnetic fields (EMF) are carcinogenic to humans and classified them as possibly carcinogenic to humans class 2B [1]. This challenges existing exposure limits for general public's health protection [2] [3] and further enhances existing public concern about brain cancer risk from mobile phones.

In the RF range the situation regarding epidemiologic studies is characterized by two opposing positions. Several studies from the Swedish group of Hardell et al. (SHG) report on consistent elevated risks of brain cancer [4]-[9] which opposes the numerous other studies, including the multinational Interphone study (IPS) with 13 countries participating [10], and all other national studies (ONS) including Sweden, which report on a lack of carcinogenic evidence up to 10 years mobile phone use but conclude there is some uncertainty with regard to specific cancers such as glioma at prolonged exposure [11]-[25]. This raised further concern and increased the need for clarification.

In this study it is shown that conclusions on suspected long-term risks are possible by a synoptic view on the existing body of evidence, because fortunately, it has become common practice that papers on epidemiologic studies include tables with extensive numbers of detailed results in terms of risk estimates (odds ratios, OR) which have been calculated for a large variety of different scenarios such as kind of tumor, tumor location, laterality, radiation source (e.g. mobile phone, wireless, DECT), region (e.g. urban or rural), magnitude of exposure and dose metric (e.g. subscription time, use time, latency time, regular use, cumulated use time cumulated call time, cumulated number of calls). However, interestingly, so far authors as well as reviewers including risk assessment bodies draw their conclusions based on only one or few selected (usually worst-case) OR values per study [1] [24] [25]. So far, little use has been made of the entire pool of available data.

Therefore, in a new approach of synoptic analysis of all ORs published so far, a puzzling fact could be identified. In contrast to the ELF range, where the ORs reported with childhood leukemia fitted well into one common data pool [10], a quite different scenario was found in the RF range [11]: The synoptic analysis identified a striking difference between reported results of mobile phone brain cancer studies of one particular research group, namely the SHG group which exhibits an implausible pattern compared to all other data, namely of IPS and ONS [13]-[25] which were consistent within themselves. This indicated major differences in SHG's studies with regard to methodology and/or data analysis. With the synoptic analysis it could be already shown that ORs critically depend on the underlying number of exposed cases [10] [11]. Moreover, surprisingly, in spite of the puzzling contradictions and the different data patterns, with increased number of exposed cases the data pools on brain cancer of both groups, namely of SHG as well as of all other studies (IPS and ONS) headed towards the same OR value-although from different sides of the zero-risk line. Even more, the ORs converged towards a reduced risk $(\mathrm{OR}=0.8)$ and, hence, were reassuring rather than alarming [11]. However, since this synoptic analysis did not yet differentiate between various types of brain cancer, it could be argued that existent risks of particular cancer types could have been masked by lacking risks of others. Therefore, this paper presents the results of the analysis on a specific brain cancer type, namely on glioma, with particular emphasis on the suspected long-term risk.

\section{Method}

In the new synoptic approach all reported ORs of available epidemiologic studies on mobile phone use and risk of glioma published from 2001 until 2014 were pooled and analyzed. Studies were identified by a literature search in the data bases Pubmed, FEMU and IEEE Xplore. The studies were included without further selection such as with regard to size of a study or exposure metric. None of the available studies was rejected. Therefore, the entire body of available data was analyzed without any further restrictions. In case ORs were given both unadjusted and adjusted for cofactors, the latter were used.

The synoptic analysis should allow getting an overview on the existing body of evidence, identifying potential discrepancies and outliers, finding dependencies on parameters, if existent, and testing the null hypothesis. Consequently, the pool of data was to investigate in particular with regard to:

1) the null hypothesis that there is no causal relationship between mobile phone use and glioma;

2) a potential dependence of ORs on the number of exposed cases $N_{\exp }$ used for OR calculation as surrogate for the statistical power;

3) a potential dependence of ORs on the accumulated exposure (dose) in particular with regard to a potential long-term glioma risk. Since studies report on different estimated dose metrics such as cumulated use time 
(CUT), cumulated call time (CCT) and cumulated number of calls (CNC), analyses were made on each of them.

Usually, epidemiological studies use quite different cut-off points to classify the accumulated exposure (dose). For synoptic analysis this allowed generating various data points to quantitatively analyze a potential dose dependence rather than comparing "heavy users" (which are differently defined among the studies) with others. However, the dose response analysis does not account for the different statistical power of the various ORs. Therefore, in addition to time trend analyses, the data were also separated into different data pools, related to low and high dose, respectively, to account for the different statistical power and check whether ORs in dependence on $\mathrm{N}_{\mathrm{exp}}$ converged, and if so, towards which endpoints.

\section{Results}

Overall, 16 epidemiologic studies on mobile phone use and glioma with 523 reported OR values could be analyzed. (Please note that due to overlap of similar OR values, not all data points can be identified in the presented figures.) Figure 1 presents an overview on the pooled data of all glioma studies of SHG as well as of ONS and IPS. It can be seen that ORs and their variance clearly depend on the number of exposed cases $\mathrm{N}_{\exp }$ which was already encountered in the pooled brain cancer data [11]. Like the overall brain cancer data also glioma data of ONS and IPS exhibit the well-known funnel-shaped pattern with a wide variance extending to either side of the zero risk line $\mathrm{OR}=1$ at low $\mathrm{N}_{\mathrm{exp}}$, comprising three orders of magnitudes from $\mathrm{OR}=0.3$ up to $\mathrm{OR}=30$. It can be seen that there are some outliers (related to results from [13]). With increasing $\mathrm{N}_{\exp }$ the variance decreases and ORs become lower and lower, and finally converge towards $\mathrm{OR}=0.8$ which indicates a reduced risk.

In contrast to this and similar to brain cancer results SHG data considerably differ from all other data with regard to their variance and the data pattern. The overwhelming majority of ORs is larger than 1 , hence ORs are at only one side of the zero risk line ( $\mathrm{OR}=1)$ which is not plausible from a statistical point of view. Consequently, the majority is indicating an increased risk (Figure 1). However, it is interesting to note, that in spite of the puzzling pattern, with increasing $\mathrm{N}_{\exp }$ also SHG data converge to the same endpoint as ONS and IPS namely towards reduced risk. However, with regard to the obvious methodological difference and bias, SHG data had to be excluded from the following analysis of a potential dose-response.

Since Figure 1 includes all glioma data irrespective the duration or intensity of mobile phone use, it does not yet allow concluding on any dose response. Therefore, this was analyzed in a second step based on different dose surrogates. Figure 2 shows the time trend of pooled ORs of IPS and ONS in dependence on the cumulated use years (CUY) as derived from questionnaires in terms of years of use or latency time until diagnosis. The quadratic regression curve across the time trend data remains mostly below the zero-risk line $(\mathrm{OR}=1)$ and only beyond 10 years approaches zero risk $(\mathrm{OR}=1)$. The quadratic regression coefficient amounts to 0.028 . Therefore, in spite of the reassuring results up to 10 years it could be argued that the final slight increase towards OR $=1$ could indicate a potential long-term effect. However, the time trend suffers from a major disadvantage, namely, that it gives the same weight to all ORs irrespective their statistical power $\left(\mathrm{N}_{\exp }\right)$. To account for this, the entire OR data pool was separated into two dose-related sub-pools, one with CUY lower than 5 years and another with CUY larger or equal 10 years (Figure 3). Although the number of data points is lower, it can be clearly seen that both sub-pools exhibit a quite similar pattern. In both cases with increasing $\mathrm{N}_{\exp }$ the risk estimates decrease. None of the sub-pools tends towards an increased risk. On contrary, they converge towards reassuring endpoints ( $\mathrm{OR}=0.8$ and $\mathrm{OR}=1$, respectively).

The same was done with another surrogate of cumulated exposure, namely the (estimated) cumulated call time (CCT). For its calculation, three estimates are necessary, namely user's estimates on their use time, their average number of calls and their average call time. Similar to CUY, the time trend exhibits no increased risk, however, again ORs increase towards the largest CCT value even to ORs of clearly increased risk (Figure 4). The quadratic regression coefficient amounts to 0.112 . Again, interpretation needs some caution due to the fact that the same weight is given to all OR values irrespective their statistical power. Therefore, the data pool was again separated into two sub-pools with CCTs lower and higher than $500 \mathrm{~h}$, respectively. Now, similar to CUY in dependence of $N_{\exp }$ the patterns of both sub-pools clarifies the seeming long-term effect: Both patterns show the well-known decrease of ORs with increasing statistical power $\left(\mathrm{N}_{\text {exp }}\right)$. Again, none of the sub-pools tends towards an increased risk (Figure 5). Now, the seemingly increased risk at highest CCT as indicated by the time trend can be explained. It is caused by the high variance and some offset of ORs at low $\mathrm{N}_{\exp }$ rather by a long-term effect. 



Figure 1. Reported odds ratios (OR) of epidemiological case-control studies on glioma and mobile phone use over the associated number of exposed cases $\mathrm{N}_{\text {exp }}$; data of the Swedish Hardell group (above) and results of all other studies (below); diamonds: Swedish Hardell studies, grey points: all other national studies; black points: interphone study.

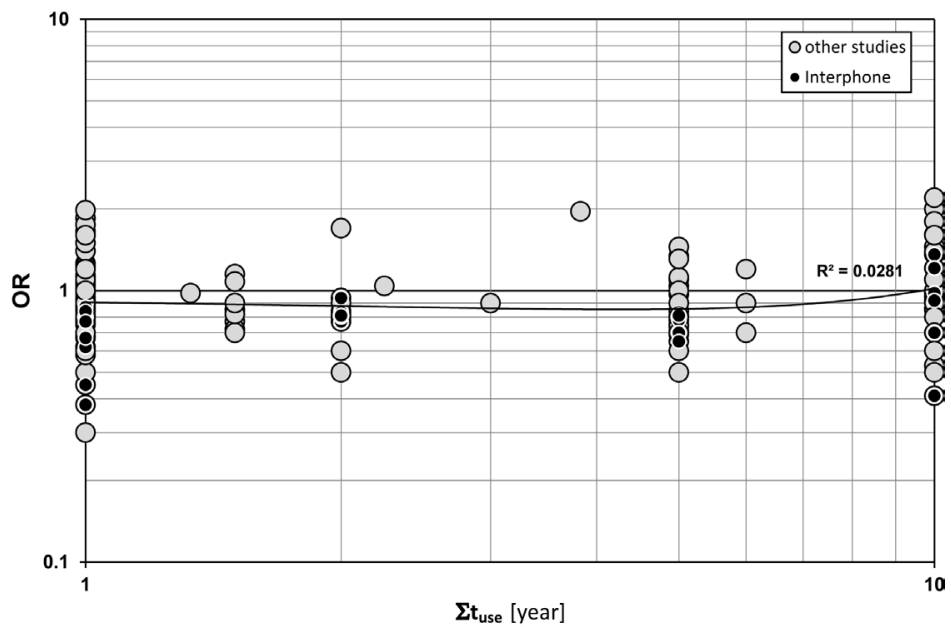

Figure 2. Reported odds ratios (OR) of epidemiological case-control studies on glioma and mobile phone use (without SHG data) over the cumulated use time $t_{\text {use }}$, grey points: all other national studies; black points: interphone study. 

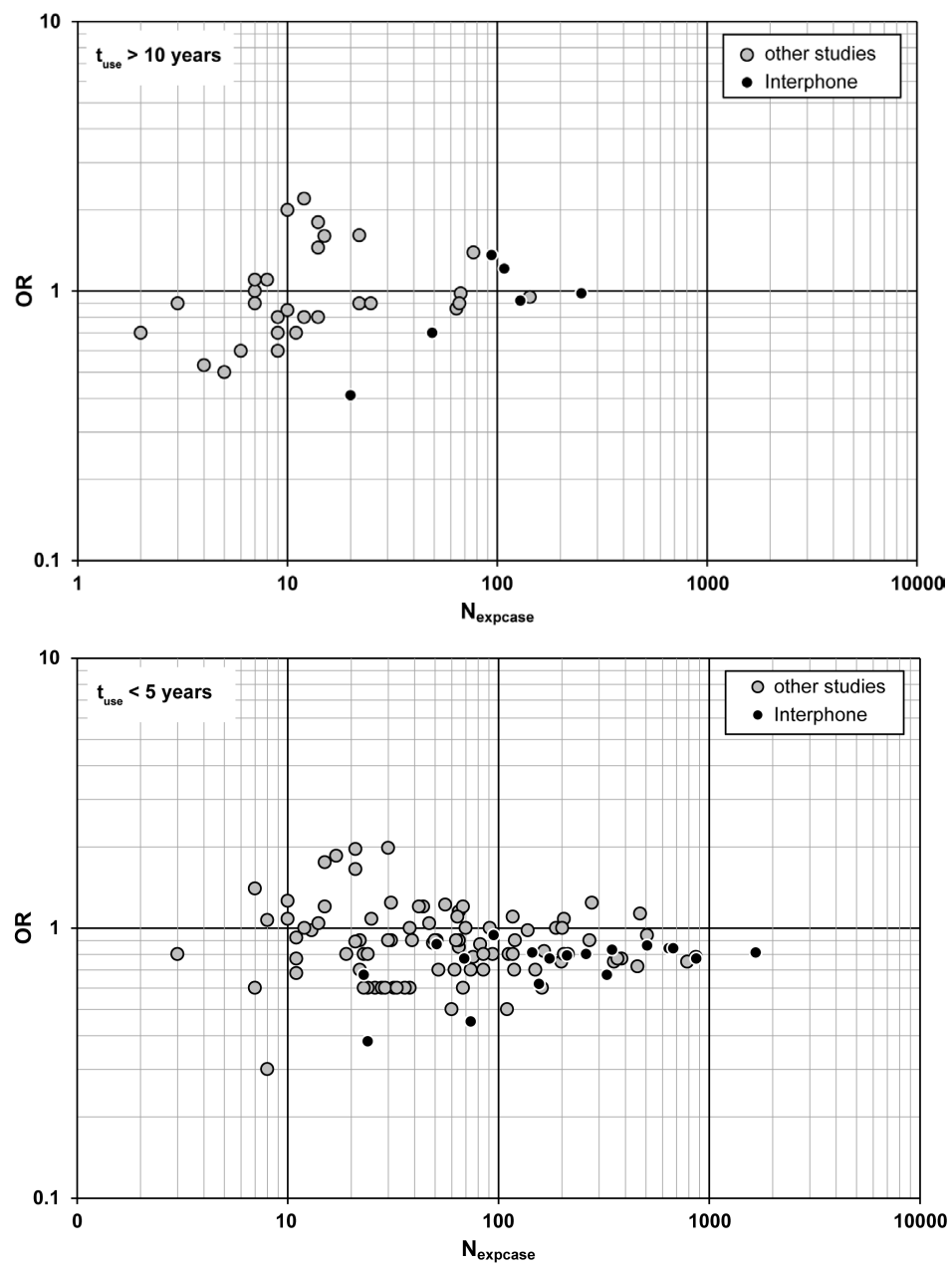

Figure 3. Reported odds ratios (OR) of epidemiological case-control studies on glioma and mobile phone use (without SHG data) over the associated number of exposed cases $\mathrm{N}_{\text {exp }}$; use time larger than 10 years (above), use time larger lower than 5 years (below); grey points: all other national studies; black points: interphone study.



Figure 4. Reported odds ratios (OR) of epidemiological case-control studies on glioma and mobile phone use (without SHG data) over the cumulated call time $t_{\text {call }}$; grey points: all other national studies; black points: interphone study. 

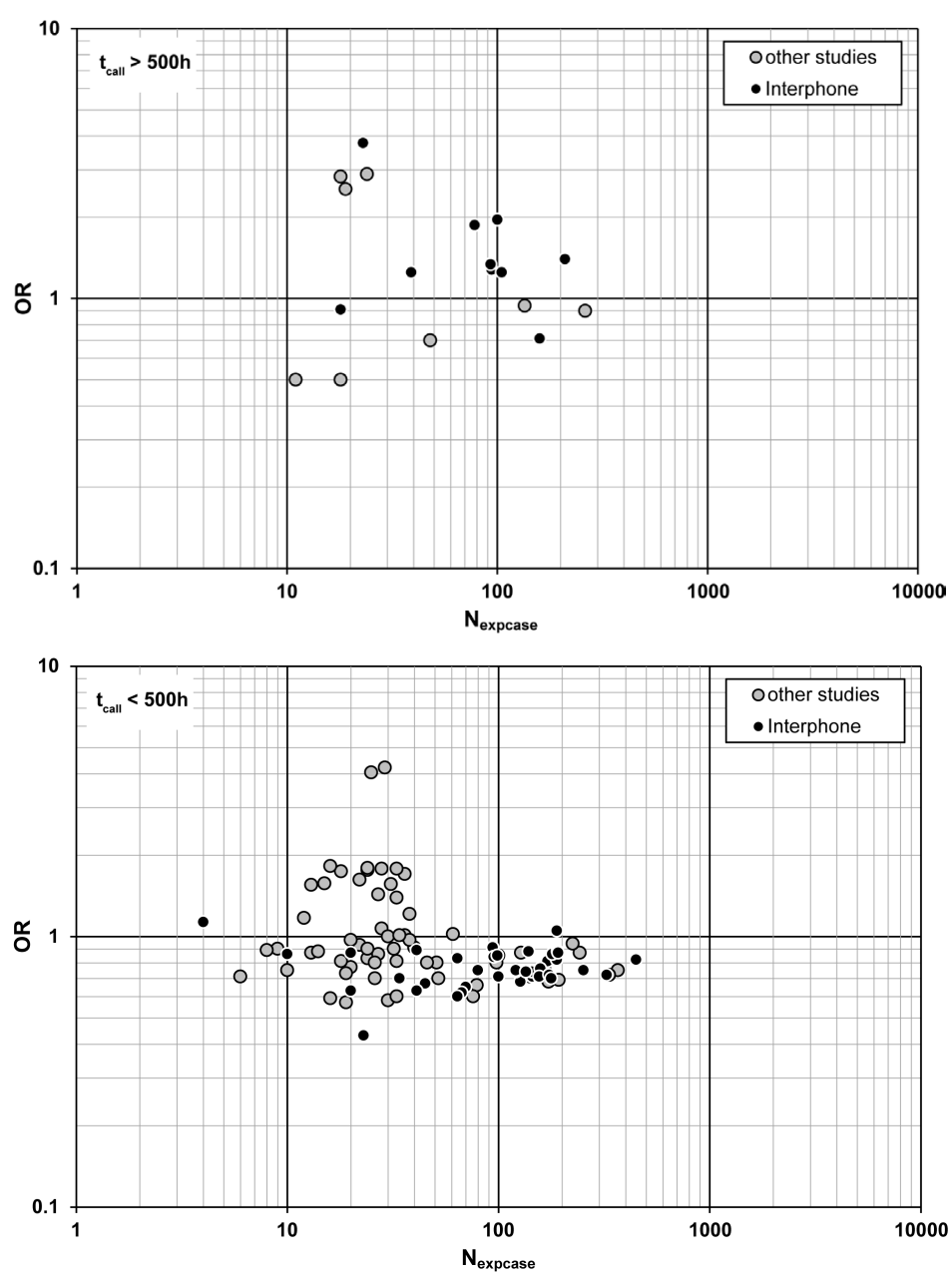

Figure 5. Reported odds ratios (OR) of epidemiological case-control studies on glioma and mobile phone use (without SHG data) over the associated number of exposed cases $\mathrm{N}_{\text {exp }}$ with cumulated call time larger than $500 \mathrm{~h}$ (above) and lower than $500 \mathrm{~h}$ (below); grey points: all other national studies; black points: interphone study.

The analysis of the third dose surrogate, the cumulated number of calls (CNC) as derived from questionnaires also confirmed the previous results (Figure 6). The time trend regression line stays in the reduced risk region at $\mathrm{OR}=0.83$ until about 8000 calls and increases to $\mathrm{OR}=1$ towards the highest number of calls. The check of the impact of low $\mathrm{N}_{\text {exp }}$ was made with two sub-pools of high and low number of calls, namely with $\mathrm{N}_{\text {call }}>10,000$ and $\mathrm{N}_{\text {call }}<5000$, respectively. In agreement with the results of the other dose surrogates the patterns exhibited the well-known funnel-shaped data distribution with high OR variance at low $\mathrm{N}_{\exp }$ and again with a trend towards reduced risk both for results with high and low number of calls. Again, this does not indicate an increased risk of heavy and/or long-term users (Figure 7).

\section{Discussion}

It is interesting to note that reported risk estimates receive different public and scientific attention, depending on whether they indicate increased or decreased risk. This is of particular importance with mobile phone use and glioma, where the consistent OR values below 1 are readily interpreted as an effect of bias rather than an EMF effect just because protective effects were postulated as "implausible" while harmful effects seemingly are not. This is in spite of the fact that there are no established mechanisms for either assumption. Such a "plausibility bias" explains why for most dose estimates consistent results with ORs lower 1 indicating reduced risks gain little attention while the single OR above 1 for estimated "heavy" users dominates risk perception-in spite of the 




Figure 6. Reported odds ratios (OR) of epidemiological case-control studies on glioma and mobile phone use (without SHG data) over the cumulated number of calls $\mathrm{N}_{\text {call }}$; grey points: all other national studies; black points: interphone study.


Figure 7. Reported odds ratios (OR) of epidemiological case-control studies on glioma and mobile phone use (without SHG data) over the associated number of exposed cases $\mathrm{N}_{\exp }$ with cumulated numbers of calls larger than 10,000 (above) and lower than 5000 (below); grey points: all other national studies; black points: interphone study. 
fact that it is most prone to uncertainties such as due to unreliable exposure assessment in the presence of technological changes over the years, unknown background exposure, inadequate and even implausible dose estimates, different recall bias of cases (or their proxis) and controls, in addition, there are biased selection of cases, biased participation rates, insufficient assessment of individual cancer-related confounders and different criteria used in studies for classifying exposure groups.

It is well known but needs to be repeated that risk estimates of epidemiological studies indicate just an association rather than a proof of causality. Whatever the OR value may be, whether there is a causal relationship needs to be proven in a second step. Consequently, ORs indicating a reduced glioma risk are not implausible by themselves. Not believing does not mean not existing. Therefore, analysis should be open for any outcome and likewise for ORs higher than and lower than 1. Risk estimates below 1 do not justify à priory negligence but may be explained also by other causes than methodological bias such as lifestyle, increased brain activity and, hence, cerebral blood flow during a call, yet unidentified co-factors-or others including potential subtle beneficial thermal EMF effects.

In spite of many ORs lower 1 there is concern about a potential long-term risk. This concern is mainly driven by controversial results of the SHG versus all other national and international studies and further supported by the (relative) OR increase at suspected long-term and/or heavy users irrespective the fact that even in this case ORs may stay at or below 1, and irrespective the fact that long-term results suffer from the highest uncertainty and the highest probability of bias. So far, by opposing studies with different outcome (and conclusions) the question whether or not a long-term health risk exists could not be convincingly answered.

The new approach to synoptically look at all available data was triggered by the common habit of epidemiologists, to publish tables over tables with ORs (some papers report over 100 individual ORs) without discussing these results in detail. Frequently, this detailed information (which is welcome as such) remains ignored, even if it contains statistical significant results. Usually only one or few ORs are picked out of the published bulk of data (and/or the pool of unpublished results) and are presented as "the" result without any convincing rational for the particular selection. Even in reviews epidemiologic studies are characterized by only one (or few) "representative” OR. At the end, without convincing reasoning it is concluded whether there is a risk or not. In addition, this (common) kind of reviewing suffers from another major flaw: In concentrating on selected ORs from different studies, each of the study-representing ORs is given the same weight-in spite of different number of exposed cases and hence, different statistical power. It is no surprise that this necessarily leads to conclusions that may be challenged.

The new synoptic approach overcomes these deficiencies and accounts for the different reliability of calculated ORs. It allows bringing the various published ORs into perspective. It also makes it possible to clarify the seeming increase of OR with dose and identifying it as an effect of the low number of associated exposed cases. It is no surprise that heavy users are less numerous than average users and, consequently, with low $\mathrm{N}_{\exp }$ the uncertainty of associated ORs is increased. It is not self-evident and important to note, that with increasing $\mathrm{N}_{\text {exp }}$ ORs tend towards a final endpoint, in particular indicating no or even reduced risk. It is interesting that data exhibit the same trend also at every dose surrogate irrespective their different reliability and different meaning for example, high number of calls can be accumulated by short-term intense use as well as long-term occasional use. The reliability of dose surrogates differs. On the one hand, CUY (cumulated use years) might be assessed with more reliability because it may be based on hard facts such as subscriber's contracts (although this does not yet allow differentiating the intensity of use). On the other hand, CNC (cumulated number of calls) requires a guess about the average number of calls over many years while CCT (cumulated call time) needs a further guess about the average duration per call. The limited reliability of dose data is demonstrated by the existence of obvious implausible guesses (such as $12 \mathrm{~h}$ call time per day) as demonstrated in the Interphone study.

The synoptic approach and the use of every reported OR irrespective the quality of a study could be challenged by arguments such as:

- Pooling studies with quite different methodology is not justified. This argument ignores that the synoptic approach must not be confused with meta-analyses. While these have their merit on their own, the joint analysis of studies with the same design still stays with their methodological weaknesses and biases. In contrast, in the presence of a body of data a synoptic analysis is just what scientist do in a first step, namely, to get a general overview over the available evidence. In a second step, it allows checking consistency and whether the data patterns provide additional insight—or not. After decades of epidemiological studies and a meanwhile huge amount of available data, a "more of the same” strategy would hardly contribute to new insights. 
The existent body of information allows already concluding that new studies with the same design adding some new data points to the pool of existent data will hardly change the overall image.

- Pooling of ORs from studies with different methodology does not make sense. In fact, the contrary is true. It allows concluding on the consistency of the data and identifying inconclusive results, if there are any. The benefit of the synoptic view could already be demonstrated very clearly [10] [11]. If all epidemiological studies were reliable, in spite of existing variance in the results, overall, the results should fit in a consistent pattern. It was surprising how clearly it was possible to identify the puzzeling SHG cluster at first glance, which is just neither plausible by itself, nor plausible compared to all the other studies (Figure 1). Such a situation was not found in the ELF range after 36 years of epidemiological research [10].

- Pooling ORs of different exposure scenarios and dose parameters is no valid approach. This argument ignores, that of course sub-pooling can be done (and was done) also with data related to different scenarios, in addition to the overall pooled data (see Figures 2-7). In addition, the overall synoptic approach is very useful for testing the null hypothesis: If there is no causal relationship, different exposure scenarios or exposure metrics would not count and consequently, the related data would fit into a common and consistent pattern converging towards a final value. If not, the associated data patterns would differ in some way or another and convergence, if seen, may be towards a risk estimate unequal 1. In fact, with regard to glioma, the pattern(s) confirm the null hypothesis: Even when data were analyzed separately and in dependence of dose, the resulting patters still agreed fairly well.

- Pooling of studies of different quality degrades the conclusions. In fact, weighing the evidence by the quality of studies and excluding inadequate studies from analysis is a common and valuable approach in risk assessment. However, it suffers from the fact that it makes it necessary to justify the dismissal and acceptance of studies which may be challenged by stakeholders. In addition, selection may be prone to bias. This is demonstrated by the fact that different assessment groups such as ICNIRP and WHO versus the Bioinitiative group give quite different weight to the same studies. Therefore, exclusion of studies feeds the public controversy between "believers" and "non-believers" of mobile phone health risks. In contrast to this, the synoptic approach does not need selection and, hence does not need justifying any selection criterion. Even if single studies may not be accessible, this would not degrade the outcome in the presence of a sufficient data pool.

- Not excluding dubious studies might impair the conclusion. This argument does not hold, since few additional data points will hardly change the overall pattern. In addition, the quality criteria of research funds are such that the design of (expensive) large studies is reviewed very critically while small studies may not have been assessed at all but usually involve small numbers of cases and, hence report on ORs in an $\mathrm{N}_{\text {exp }}$ range with high variance and do not critically influence the synoptic analysis. Furthermore, outliers can easily be identified by their implausibility compared to the body of evidence. It must be noted that implausible ORs are not restricted to poor studies only. Even reputed studies may include some implausible ORs such as presented in Figure 1 with two OR outliers at $\mathrm{N}_{\text {exp }}=140$.

- Multiple papers of the same study generate disproportionate numbers of ORs and hence may degrade the outcome. Fortunately, this argument does not hold. In fact, papers of the same basic data report on similar ORs. Consequently, such ORs overlap, hence, do not change the pattern. (Overlap is the reason why not all analyzed data points can be identified in the presented figures.) Results, that do not fit in the overall cluster can be identified and checked for consistency and plausibility (such as the SHG data, Figure 1).

The detailed analysis of the glioma data showed, that irrespective their inherent differences none of the various dose parameters did indicate an increased risk if the statistical power of ORs (indicated by $\mathrm{N}_{\mathrm{exp}}$ ) was taken into account. Furthermore, it allowed identifying an obvious bias in the SHG data and an their implausible distribution. However, it could be demonstrated that at the end with increasing $\mathrm{N}_{\exp }$ even SHG data finally converge towards the same finding, namely lack of increased glioma risk.

Hopefully the clarification by synoptic analysis can contribute to a less emotional debate and the acceptance of the fact that overall, with regard to mobile phone and glioma risk the available evidence is reassuring rather than alarming.

\section{Conclusions}

This synoptic analysis of all available epidemiological data on mobile phone use and glioma risk allows the fol- 
lowing conclusions:

Similar to the overall brain cancer results [26]-[28] glioma data allow identifying two quite different data pools, one reported from one single group only with alarming results however, with an implausible pattern and an obvious offset which indicates a methodological difference rather than a biological effect. Interestingly, with increasing statistical power even this data pool converges towards lack of increased risk.

The analysis of different dose-related parameters and the pattern of all pooled data do not support the assumption of a glioma risk of long-term and/or heavy mobile phone users.

With increasing statistical power the risk estimates converge towards a reduced health risk. However, in lack of a convincing EMF interaction mechanism the indication of a reduced health risk estimate does not justify concluding on a causal relationship with EMF and a beneficial effect either.

Apart from differences in detail, a look on all available data including SHG, IPS and ONS, consistently does not indicate an increased mobile phone glioma risk, neither short-term nor long-term. The available evidence is reassuring rather than alarming.

\section{Conflict of Interest}

The author declares no conflict of interest.

\section{References}

[1] IARC (2011) IARC Monographs on the Evaluation of Carcinogenic Risks to Humans. Volume 102. Non-Ionizing Radiation, Part 2: Radio Frequency (RF) Electromagnetic Fields. Lyon, 2011.

[2] ICNIRP (1998) Guidelines for Limiting Exposure to Time-Varying Electric, Magnetic and Electromagnetic Fields (up to $300 \mathrm{GHz})$. Health Physics, 74, 494-522.

[3] IEEE (2005) IEEE Standard for Safety Levels with Respect to Human Exposure to Radio Frequency Electromagnetic Fields (up to $300 \mathrm{GHz}$ ).

[4] Hardell, L. and Carlberg, M. (2013) Using the Hill Viewpoints from 1965 for Evaluating Strengths of Evidence of the Risk for Brain Tumors Associated with the Use of Mobile and Cordless Phones. Reviews on Environmental Health, 28, 97-106. http://dx.doi.org/10.1515/reveh-2013-0006

[5] Hardell, L., Carlberg, M., Söderqvist, F. and Hansson Mild, K. (2013) Case-Control Study of the Association between Malignant Brain Tumors between 2007 and 2009 and Mobile and Cordless Phone Use. International Journal of Oncology, 43, 1833-1845.

[6] Carlberg, M., Hansson Mild, K. and Hardell, L. (2013) Use of Mobile Phones and Cordless Phones Is Associated with Increased Risk for Glioma and Acoustic Neuroma. Pathophysiology, 20, 85-110. http://dx.doi.org/10.1016/j.pathophys.2012.11.001

[7] Hardell, L., Carlberg, M. and Hansson Mild, K. (2011) Pooled Analysis of Case-Control Studies on Malignant Brain Tumors and the Use of Mobile and Cordless Phones and Deceased Subjects. International Journal of Oncology, 38, 1465-1474. http://dx.doi.org/10.3892/ijo.2011.947

[8] Hardell, L., Carlberg, M. and Hansson Mild, K. (2006) Pooled Analysis of Two Case-Control Studies on Use of Cellular and Cordless Telephones and the Risk for Malignant Brain Tumors Diagnosed in 1997-2003. International Archives of Occupational and Environmental Health, 79, 630-639.

[9] Hardell, L., Carlberg, M. and Hansson Mild, K. (2005) Use of Cellular Telephones and Brain Tumor Risk in Urban and Rural Areas. Occupational and Environmental Medicine, 62, 390-394. http://dx.doi.org/10.1136/oem.2004.017434

[10] Leitgeb, N. (2014) Childhood Leukemia Not Linked with ELF Magnetic Fields. Journal of Electric and Magnetic Analysis and Applications, 6, 174-183. http://dx.doi.org/10.4236/jemaa.2014.67017

[11] Leitgeb, N. (2014) Synoptic Analysis of Epidemiologic Evidence of Brain Cancer Risks from Mobile Communication. JEMAA, 6, 413-424.

[12] Interphone Study Group (2010) Brain Tumor Risk in Relation to Mobile Telephone Use: Results of the INTERPHONE International Case-Control Study. International Journal of Epidemiology, 39, 675-694. http://dx.doi.org/10.1093/ije/dyq079

[13] Coureau, G., Bouvier, G., Lebailly, P., Fabbro-Peray, P., Gruber, A., Leffondre, K., Guillamo J.-S., Loiseau, H., Mathoulin-Péklissier, S., Salamon, R. and Baldi, I. (2014) Mobile Phone Use and Brain Tumors in the CERENAT CaseControl Study. Occupational and Environmental Medicine, 71, 514-522. http://dx.doi.org/10.1136/oemed-2013-101754

[14] Swerdlow, A.J., Feychting, M., Green, A.C., Kheifets, L. and Savitz, D. (2011) Mobile Phones, Brain Tumors and the 
Interphone Study. Where Are We Now? Environmental Health Perspectives, 119, 1534-1538. http://dx.doi.org/10.1289/ehp.1103693

[15] Takebayashi, T., Varsier, N., Kikuchi, Y., Wake, K., Taki, M., Watanabe, S., Akiba, S. and Yamaguchi, N. (2008) Mobile Phone Use, Exposure to Radiofrequency Electromagnetic Field, and Brain Tumor. A Case-Control Study. British Journal of Cancer, 98, 652-659. http://dx.doi.org/10.1038/sj.bjc.6604214

[16] Hours, M., Bernardt, M., Montestrucq, L., Arslan, M., Bergerer, A., Deltour, I. and Cardis, E. (2007) Cell Phones and Risk of Brain and Acoustic Nerve Tumors: The French INTERPHONE Case-Control Study. Revue d'Epidémiologie et de Santé Publique, 55, 321-332.

[17] Schlehofer, B., Schlaefer, K., Blettner, M., Berg, G., Böhler, E., Hetinger, I., Kunna-Grass, K., Wahrendorf, J. and Schüz, J. (2007) Environmental Risk Factors for Sporadic Acoustic Neuroma (Interphone Study Group Germany). European Journal of Cancer, 43, 1741-1747. http://dx.doi.org/10.1016/j.ejca.2007.05.008

[18] Hepworth, S.J., Schoemaker, M.J., Muir, K.R., Swerdlow, A.J., van Tongeren, M.J. and McKinney, P.A. (2006) Mobile Phone Use and Risk of Glioma in Adults: Case-Control Study. BMJ, 332, 883-887. http://dx.doi.org/10.1136/bmj.38720.687975.55

[19] Klaeboe, L., Blaasaas, K.G. and Tynes, T. (2006) Use of Mobile Phones in Norway and Risk of Intracranial Tumors. European Journal of Cancer Prevention, 16, 158-164.

[20] Lahkola, A., Auvinen, A., Raitanen, J., Schoemaker, M.J., Christensen, H.C., Feychting, M., Johansen, C., Klaeboe, L., Lönn, S., Swerdlow, A.J., Tynes, T. and Salminen, T. (2006) Mobile Phones Use and Risk of Glioma in 5 North European Countries. International Journal of Cancer, 120, 1769-1775.

[21] Lönn, S., Ahlbom, A., Christensen, H.C., Johansen, C., Schüz, J., Edström, S., Henrikksson, G., Lundgren, J., Wennerberbg, J. and Feychting, M. (2006) Mobile Phone Use and Risk of Parotid Gland Tumor. American Journal of Epidemiology, 164, 637-643. http://dx.doi.org/10.1093/aje/kwj242

[22] Schüz, J., Böhler, E., Berg, G., Schlehofer, B., Hettinger, I., Schlaefer, K., Wahrendorf, J., Kunna-Grass, K. and Blettner, M. (2006) Cellular Phones, Cordless Phones and the Risk of Glioma and Meningioma (Interphone Study Group Germany). American Journal of Epidemiology, 163, 512-520.

[23] Lönn, S., Ahlbom, A., Hall, P. and Feychting, M. (2005) Long-Term Mobile Phone Use and Brain Tumor Risk. American Journal of Epidemiology, 161, 526-535. http://dx.doi.org/10.1093/aje/kwi091

[24] Auvinen, A., Hietanen, M., Luukkonen, R. and Koskela, R.S. (2002) Brain Tumors and Salivary Gland Cancers among Cellular Telephone Users. Epidemiology, 13, 356-359. http://dx.doi.org/10.1097/00001648-200205000-00018

[25] Inskip, P.D., Tarone, R., Hatch, E., Wilcosky, T.C., Sjapiro, W.R., Selker, R.G., Fine, H.A., Black, P.M., Loeffler, J.S. and Kinet, M.S. (2001) Cellular Telephone Use and Brain Tumors. New England Journal of Medicine, 34, 79-86. http://dx.doi.org/10.1056/NEJM200101113440201

[26] Ahlbom, A., Feychting, M., Green, A., Kheifets, L., Savitz, D.A. and Swerdlow, A.J. (2009) Epidemiologic Evidence on Mobile Phone and Tumor Risk: A Review. Epidemiology, 20, 639-652. http://dx.doi.org/10.1097/EDE.0b013e3181b0927d

[27] Kundi, M. (2009) The Controversy about a Possible Relationship between Mobile Phone Use and Cancer. Environmental Health Perspectives, 117, 306-324. http://dx.doi.org/10.1289/ehp.11902

[28] Lagorio, S. and Röösli, M. (2014) Mobile Phone Use and Risk of Intracranial Tumors: A Consistency Analysis. Bioelectromagnetics, 35, 79-90. http://dx.doi.org/10.1002/bem.21829 